\title{
Practical Aspects of Performing Quantitive EELS Measurements of Gas Compositions in Closed-Cell Gas Reaction S/TEM
}

Kinga Unocic ${ }^{1}$, Michael Griffin ${ }^{2}$, Joshua Schaidle ${ }^{2}$, Susan Habas ${ }^{2}$, Franklin Walden ${ }^{3}$, Raymond Unocic ${ }^{1}$ and Lawrence Allard ${ }^{4}$

${ }^{1}$ Oak Ridge National Laboratory, United States, ${ }^{2}$ National Renewable Energy Laboratory, United States, ${ }^{3}$ Protochips Inc., United States, ${ }^{4}$ UT-Battelle LLC, Oak Ridge, Tennessee, United States

In situ electron microscopy is an attractive method that has been extensively used in dynamic studies of a variety of material systems. Both environmental transmission electron microscopy (E-TEM) and in situ closed-cell gas reaction (CCGR) scanning transmission electron microscopy (S/TEM) have their own advantages and disadvantages. For example, E-TEM requires a dedicated transmission electron microscope with a special vacuum system design utilizing "differential pumping" apertures in the vicinity of the sample that do not impede the electron beam, but that only allow treatments of samples at relatively low pressures $(<\sim 10$ Torr). In comparison, CCGR-STEM requires only a dedicated heating holder incorporating a system to deliver gases into a cell defined by thin amorphous SiN "windows" through which the electron beam passes; this geometry allows the CCGR holder to operate up to atmospheric pressure. Previously, it has been shown that detection and compositional analysis of flowing gases in ETEM is possible via electron energy loss spectroscopy (EELS) (Fig. 1a) [1,2], where the electron beam "sees" the gas atmosphere through a path length of $\sim 1 \mathrm{~cm}$. But the electron beam in a CCGR system must pass through $\mathrm{SiN}$ membranes above and below the sample material, that affect the EELS spectrum by decreasing the signal to noise and increasing the multiple scattering that is important in the low-loss regime. Here we present measurements of flowing gases (including water vapor) at a variety of pressures in CCGR-STEM that address the challenges of this method for using EELS for gas analysis, and we show the advantages of incorporating a residual gas analysis (RGA) system on the CCGR gas exit path. In addition, we show the use of the RGA to characterize water vapor compositions, and finally we show an example of the effects of water vapor on a $\mathrm{Pt} / \mathrm{TiO}_{2}$ catalyst.

In this study, we used an FEI Titan (60-300kV) equipped with a Gatan Quantum EEL spectrometer and a JEOL 2200FS S/TEM instrument; both equipped with CEOS GmbH (Heidelberg, Ger) aberration correctors on the probe forming lenses. Both instruments are also equipped with MEMS-based CCGR systems (Protochips Atmosphere ${ }^{\mathrm{TM}}$ ), and both incorporate integrated RGA systems on the outlet side $[3,4]$. The addition of an RGA system allows operandoexperiments to be conducted to confirm the environmental conditions during in situ S/TEM examination.

A pair of microelectromechanical (MEMS)-based silicon microchip devices (i.e., an E-chip (heater) and spacer chip (bottom window chip)) that comprise the gas cell was loaded into the Atmosphere Protochips holder, initially with no sample placed on the E-chip. During in situ reaction the electron beam has to penetrate first through a $30 \mathrm{~nm}$-thick $\mathrm{Si}_{\mathrm{x}} \mathrm{N}_{\mathrm{y}}$ membrane on the E-chip, then through a 5-10 $\mu$ m-thick gap (depending upon pressure) filled with flowing or static gas(es), and finally through a $50 \mathrm{~nm}$-thick $\mathrm{Si}_{\mathrm{x}} \mathrm{N}_{\mathrm{y}}$ membrane on the window chip [3]. The gas cell was purged two times with argon, from 100 to 0.1 Torr to remove residual $\mathrm{O}_{2}$, and then with a final purging step from 100 to 0.01 Torr. EELS spectra were acquired first in vacuum through the gas cell (Fig. 1A-e): measurements were then taken under various gas conditions in the cell. The gas cell was filled with $100 \%$ Ar at 760 Torr with the E-chip kept at room temperature (RT), then the E-chip was heated to $200^{\circ} \mathrm{C}$ and EELS measurements were acquired at both conditions (Fig. B). The effect of pressure was also investigated, and EEL spectra were acquired under 
flowing Ar at $200^{\circ} \mathrm{CE}$-chip and 14.3 Torr (Fig. B-d). Subsequently, the measurements were taken under flowing helium at 760 Torr and $200^{\circ} \mathrm{CE}$-chip (Fig. 1A-b). Finally, EEL spectra under various water vapor conditions were recorded. Spectra under $100 \%$ of $\mathrm{H}_{2} \mathrm{O}$ vapor at 14.3 Torr (Fig. 1A-d) and then a mixture of $10 \%$ of water vapor and $90 \%$ of Ar at 143 Torr were acquired. Figure 2 shows an example of the effect of $\mathrm{H}_{2} \mathrm{O}$ vapor on $\mathrm{Pt}$ particle rearrangement/restructuring during exposure to flowing $\mathrm{H}_{2} \mathrm{O}$ vapor at 17 Torr and $300^{\circ} \mathrm{C}$.

In these studies, we investigated the effects of gas cell, environment, pressure, and E-chip temperature on EELS measurements of gas compositions. EELS measurements of e.g., a catalyst material and its reaction product within a CCGR system is possible, as demonstrated [5]. However, there are clear challenges in measuring static/flowing gas compositions within the gas-cell via EELS. These measurements appear to be possible for gases at high pressures (Fig. 1B-b vs. 1B-d) and within the high-loss energy range (e.g., Ar) away from plasmon tails in the EEL spectrum (Fig. 1b).Detection of gases via EELS within the lowloss energy range such as $\mathrm{He}(22 \mathrm{eV})$ or water vapor $(18 \mathrm{eV})$ is challenging due to the overlap of energy peaks with the plasmon region that derives from the SiN membrane viewing area. In summary, for in situ CCGR-STEM gas-composition measurements, it is important to have an RGA system incorporated into the experimental geometry. The CCGR system with RGA allows experiments at high pressure with accurate gas composition measurements, while the E-TEM geometry permits direct measurements of gas compositions, even in the low-loss regime, in the absence of an RGA system [6].
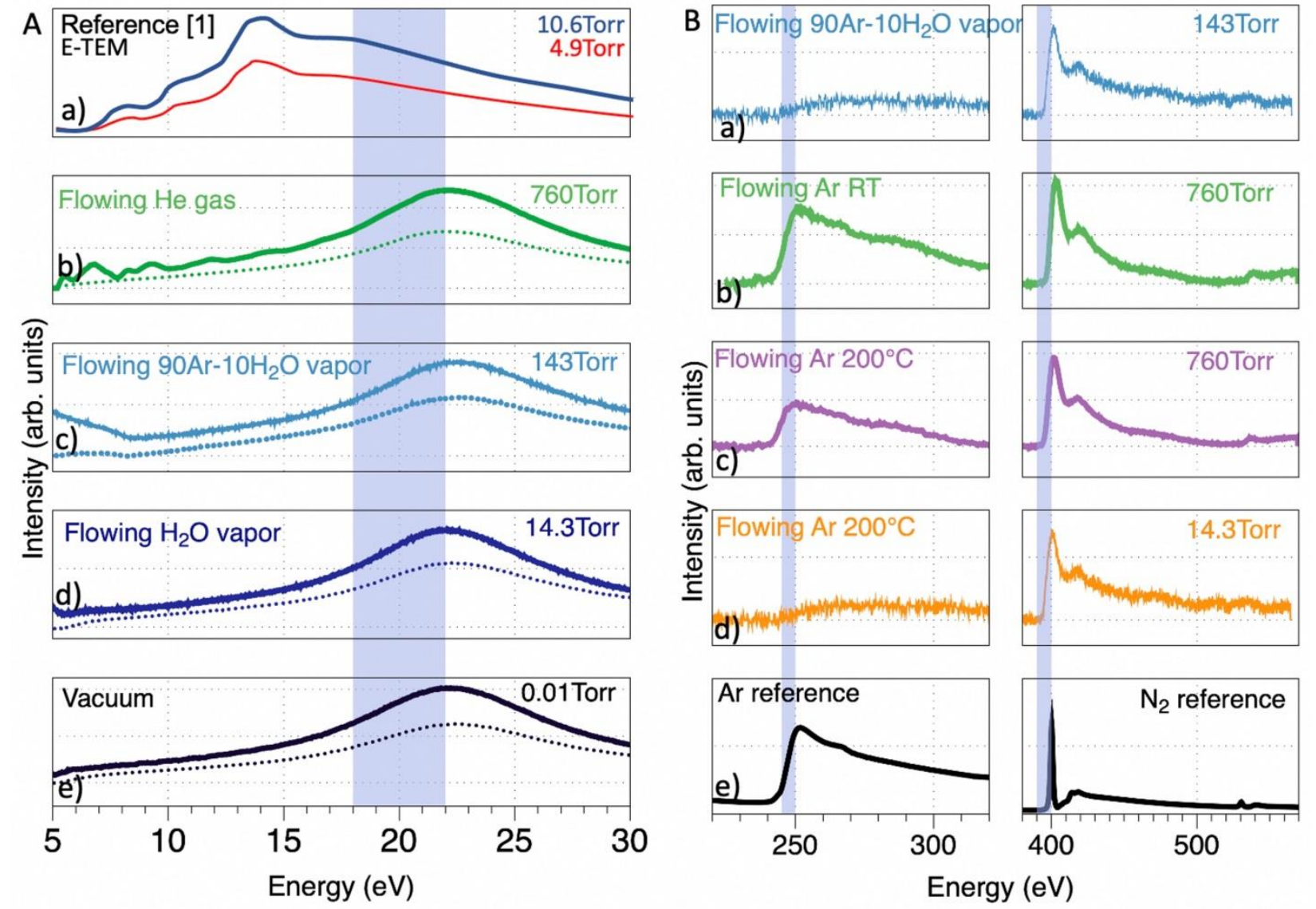

Figure 1. Figure 1. A) Low-loss EEL spectra (dotted line extracted signal, solid line deconvoluted data) and B) high-loss EEL spectra generated through an in situ CCGR set up for different indicated conditions. Note: Argon and Nitrogen reference spectra are from EELS Atlas. 

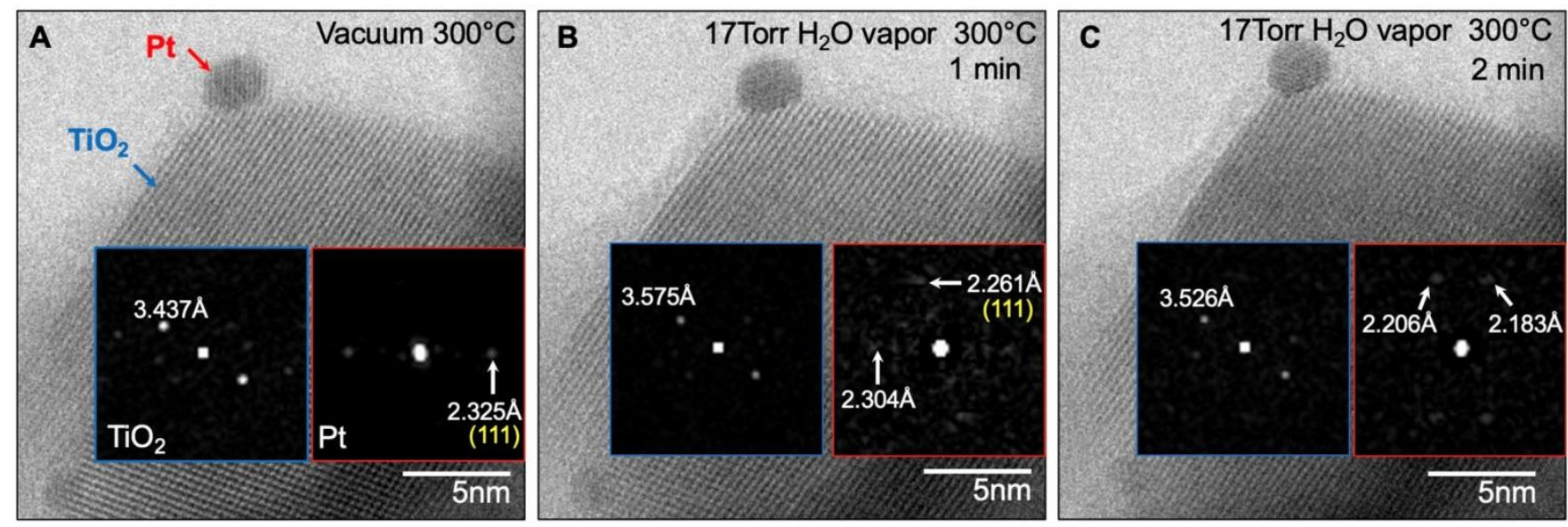

Figure 2. BF-STEM images of $\mathrm{Pt} / \mathrm{TiO} 2$ catalyst acquired under series of reaction conditions. A) After reaching $300^{\circ} \mathrm{C}$ in vacuum; After (B) $1 \mathrm{~min}$ and (C) $2 \mathrm{~min}$ exposure to flowing $\mathrm{H} 2 \mathrm{O}$ vapor at $\sim 17 \mathrm{Torr}$ at $300^{\circ} \mathrm{C}$.

\section{References}

[1] C Cassidy et al., PLOS ONE 12 (2017) p. 1.

[2] PA Crozier and S. Chenna, Ultramicroscopy 111 (2011) p. 177.

[3] KA Unocic et al., Oxid. Metals 88 (2017) p.495.

[4] KA Unocic et al., Microscopy and Microanalysis 26 (2020) p. 229.

[5] B Song et al., ACS Nano 14 (2020) p.15131.

[6] Work performed in collaboration with ChemCatBio, a member of the Energy Materials Network (EMN) and supported by the U.S. Department of Energy (DOE) Bioenergy Technology Office under Contract DE-AC0500OR22725 (ORNL), Contract DE-AC36-08-GO28308 (NREL), and Contract DE-AC02-06CH11357 (ANL). Microscopy performed as part of a user project at the Center for Nanophase Materials Sciences (CNMS), which is a U.S. DOE Office of Science User Facility. 